\title{
Similitudes y diferencias en la conceptualización de la conciencia ofrecida por el materialismo eliminativo \\ y el funcionalismo. Un análisis crítico
}




\title{
Similitudes y diferencias en la conceptualización de la conciencia ofrecida por el materialismo eliminativo y el funcionalismo. Un análisis crítico*
}

Resumen: este trabajo se dividirá en dos puntos: i) exponer las similitudes y las diferencias entre la teoría del materialismo eliminativista de los esposos Churchland y la teoría del funcionalismo de Daniel Dennett con respecto a su conceptualización sobre la concienci; y ii) analizar de manera crítica los planteamientos del materialismo eliminativista y del funcionalismo con respecto a su conceptualización acerca de la conciencia. Además, se sostendrá que en el ámbito de las ciencias cognitivas no existe un consenso, como tampoco una base teórica y conceptual sobre qué es la conciencia. Así mismo, se tendrá en cuenta que, sobre el concepto de conciencia se indaga por la pertinencia del término - si existe o no-; y de existir: si responde a patrones deterministas o si es un fenómeno ajeno a toda realidad empírica; lo cual lleva, de manera inevitable, a indagar por el debate acerca del libre albedrío.

Palabras clave: materialismo eliminativo, funcionalismo, conciencia, neurociencias, teoría memética, libre albedrío.

\section{Similarities and differences in the conceptualization of consciousness offered by eliminative materialism and functionalism. A critical analysis}

\begin{abstract}
: this work is divided into two points: i) exposes the similarities and differences between the eliminativist materialism theory of The Churchlands and Daniel Dennett's theory of functionalism; considering, in addition, the conceptualization that both theories do about consciousness; and ii) realize a critical analysis of the approaches of eliminativist materialism and functionalism with respect to its conceptualization about consciousness. In addition, it will be argued that in the field of cognitive science there is no consensus, nor a theoretical and conceptual basis on what consciousness is. Likewise, it will be taken into account that the concept of conscience will be understood by the relevance of the term -if it exists or not-; and of existing: if it responds to deterministic patterns or if it is a phenomenon alien to all empirical reality; which entails, inevitably, to investigate the debate about free will.
\end{abstract}

Keywords: eliminative materialism, functionalism, consciousness, neuroscience, memetic theory, free will.

Fecha de recepción: 5 de febrero de 2019

Fecha de aceptación: 15 de mayo de 2019

Forma de citar (APA): Esparza-Oviedo, S. M. (2020). Similitudes y diferencias en la conceptualización de la conciencia ofrecida por el materialismo eliminativo y el funcionalismo. Un análisis crítico. Revista Filosofía UIS, 19(1), DOI: 10.18273/revfil.v19n1-2020007

Forma de citar (Harvard): Esparza-Oviedo, S. M. (2020). Similitudes y diferencias en la conceptualización de la conciencia ofrecida por el materialismo eliminativo y el funcionalismo. Un análisis crítico. Revista Filosofía UIS, 19(1), 203-228.

Silvia María Esparza Oviedo: colombiana. Magíster en Filosofía de la Universidad Industrial de Santander. Profesora de la Universidad de Santander, Colombia.

ORCID iD: orcid.org/0000-0003-0584-2023

Correo electrónico: silvia.esparza.oviedo@gmail.com

"Artículo de reflexión derivado de investigación.

Revista Filosofía UIS, vol. 19 n. ${ }^{\circ}$ 1, enero - junio de 2020 


\section{Similitudes y diferencias en la conceptualización de la conciencia ofrecida por el materialismo eliminativo y el funcionalismo. Un análisis crítico'}

\section{Introducción}

Dentro del monismo psicofísico contemporáneo existen diferentes corrientes tales como: la teoría de la identidad, la teoría del materialismo eliminativo y la teoría del funcionalismo. Empecemos abordando la teoría de la identidad desarrollada a partir de los años cincuenta del siglo pasado, la cual plantea, a grandes rasgos, que los estados mentales son idénticos a los estados del cerebro. La teoría de la identidad será la precursora del materialismo eliminativo, ya que, a partir de los avances de las neurociencias se establecería una correspondencia entre los conceptos de los estados mentales y los conceptos de los estados cerebrales. De ahí que la teoría de la identidad sea considerada una de las corrientes materialistas reduccionistas.

Pasando al materialismo eliminativo, podemos decir que este es la continuidad de la teoría de la identidad, en una versión radical. Esta corriente pretende eliminar la relación de reciprocidad entre los conceptos de los estados mentales y los conceptos de los estados cerebrales para reducirse a hablar únicamente de estados cerebrales; es decir, el materialismo eliminativo toma a la neurociencia como base fundamental para explicar los procesos cerebrales, los cuales, en últimas, explican los procesos más básicos de la realidad humana.

Por su parte, el funcionalismo responde a una visión materialista noreduccionista en donde los estados mentales son comparados con los estados funcionales de las máquinas. Además, el funcionalismo, como corriente filosófica, plantea que los estados mentales y el comportamiento humano se encuentran condicionados por el ambiente. En otros términos, el funcionalismo se pregunta cómo es posible la relación de los estados mentales con la conducta y el ambiente, en el que se desenvuelve un organismo humano, haciéndose necesario el estudio causal de los fenómenos mentales, si tenemos en cuenta que, en general, todo organismo tiene una relación directa con el ambiente que lo configura (EsparzaOviedo, 2018, pp. 12-13). Así las cosas, en lo expuesto hasta el momento hemos planteado, de manera implícita, el elemento principal de la filosofía de la

${ }^{1}$ Esta es una adaptación a formato artículo de mi tesis de maestría acerca de la conceptualización de la conciencia ofrecida por la teoría del materialismo eliminativo y la teoría del funcionalismo dentro del monismo psicofísico contemporáneo, véase Esparza-Oviedo, 2018. 
mente: la problemática mente-cuerpo, el cual es considerado como un problema ontológico desde la perspectiva de Paul M. Churchland (1992), pues se trata de indagar por la existencia de algo, si tenemos claro que en el "lenguaje de los filósofos se trata de una pregunta acerca de qué cosas existen realmente y cuál es su esencia" (pp. 16-17).

Pues bien, dentro de la problemática mente-cuerpo, aquel planteamiento que nos interesa abordar es el problema de la conciencia. Sabemos que si bien el problema de la conciencia es abordado de diversas maneras en los diferentes textos de filosofía de la mente (Arias, 2016, p. 29), existen dificultades en el momento de su conceptualización, pues dentro de las ciencias cognitivas no hay un acuerdo sobre su definición: "punto crucial del debate contemporáneo en torno a la misma y, curiosamente, el acuerdo a día de hoy alcanzado respecto de dicha definición parece restringirse a la constatación de que carecemos de la misma" (Arias, 2016, p. 65).

Bien pareciera que la ciencia apunta directamente a la investigación y al resultado, más que a ofrecer definiciones, pues las definiciones se van dando con los avances mismos de la ciencia; no obstante, encontramos con bastante sospecha el hecho de ofrecer resultados y explicaciones sobre algo que propiamente no sabemos qué es, pues en el caso de las ciencias cognitivas, al no existir consenso sobre la conceptualización de la conciencia, en otras palabras, al no haber claridad sobre qué es la conciencia ${ }^{2}$, resulta sospechoso que se realicen afirmaciones contundentes como sucede con la negación del libre albedrío o la que presenta la voluntad como una ilusión. Es por ello que, en este trabajo tenemos como propósito dar cuenta de dos objetivos: i) exponer las similitudes y las diferencias entre la teoría del materialismo eliminativista de los esposos Churchland y la teoría del funcionalismo de Daniel Dennett con respecto a su conceptualización sobre la conciencia; y ii) analizar de manera crítica los planteamientos del materialismo eliminativista y del funcionalismo con respecto a su conceptualización acerca de la conciencia.

\section{Similitudes y diferencias entre el "materialismo eliminativista" y el "funcionalismo" con respecto a la conciencia}

Parece pertinente que iniciemos con la similitud más evidente, pues tanto el materialismo eliminativista como el funcionalismo son corrientes propias del materialismo psicofísico, siendo esta una de las corrientes que predominan en la actualidad dentro de la filosofía de la mente. Recordemos que, en términos

${ }^{2}$ Que en términos ontológicos nos lleva a la pregunta ¿qué es la conciencia? Es decir, los planteamientos ontológicos permiten indagar por aquello que realmente existen y cuál es su esencia (Churchland, 1992, p. 17). 
generales, el principal problema de la filosofía de la mente es el problema mentecuerpo que, como sabemos, posteriormente mutó en el problema mente-cerebro gracias al avance de las neurociencias:

Cabe en este sentido señalar que la neurociencia cognitiva trata de responder a la siguiente cuestión: ¿cómo la mente es efectivamente producida por el cerebro?, y que en su empeño por ofrecer resultados en esta dirección recurre principalmente a métodos y resultados provenientes de la neuroanatomía, la neurofisiología, la neurocirugía funcional, la neuroimagen, la psicología cognitiva y la modelización computarizada (Arias, 2012, p. 250)․․

Para complementar lo dicho, Rafael Alemañ plantea que durante el siglo XX el acelerado desarrollo de la física y de las ciencias empíricas, en general, sedujo a las demás áreas del conocimiento y, entre ellas, a las ciencias del espíritu. En consecuencia, las demás disciplinas pretendieron adquirir la misma rigurosidad y profundidad:

Cuando una ciencia triunfa tan espectacularmente en el terreno de los resultados empíricos resulta casi inevitable que sus presupuestos filosóficos, explícitos o no, acaben imponiéndose también de modo igualmente sutil. No es algo condenable en sí mismo, pero resulta problemático cuando tal aceptación se produce sin discusiones ni debates. En el caso que nos ocupa, el éxito de la física del siglo XX pareció garantizar la victoria incondicional del monismo materialista sobre cualquier otra concepción de la realidad (2013, p. 12).

Dicho esto, volvamos sobre el tema que nos interesa discurrir, que es el monismo psicofísico contemporáneo, por lo que, para facilitar nuestra comprensión, diremos que mente y cuerpo pertenecen al mismo conjunto y, en dicho conjunto, la primera entidad es ilusoria y la segunda sí tiene realidad efectiva. Además, esta explicación recoge el planteamiento del problema mente-cuerpo, de manera general, pues como sabemos dicho problema terminará por mudar en el problema mente-cerebro, en donde, a grandes rasgos, se sigue sosteniendo que todo aquello que haga referencia a una entidad mental es ilusorio, mientras que todo aquello que señale algo material —bien sea el cuerpo o, siendo más claros, el cerebro- constituye algo ciertamente real.

En consecuencia, el monismo psicofísico contemporáneo es definido como aquella corriente que reduce los procesos mentales a la materia, es decir, para el monismo materialista o fisicalista existe una sola sustancia $y$, como lo indica su nombre, es la materia misma (Bechtel, 1991, p. 127). Ahora bien, dentro del monismo materialista existen diferentes corrientes, entre las más importantes

\footnotetext{
${ }^{3}$ Con respecto a la literatura que puede ampliar este planteamiento del problema mente-cerebro, véanse Arias, 2016; Martínez-Freire, 1999; Nagel, 2000 y Velmans, 2009.
} 
están: la teoría de la identidad, el conductismo, el materialismo eliminativo y el funcionalismo. Y de estas corrientes las que nos interesan en este trabajo son el materialismo eliminativista y el funcionalismo.

Así que, sin más preámbulos, empezaremos a adentrarnos en nuestro objetivo, donde señalaremos, de manera alternada, las principales similitudes y diferencias de dichas corrientes, pues los puntos de encuentro y desencuentro nos permitirán ir avanzando hasta cumplir con nuestra meta propuesta que es, en un primer momento, ofrecer una conceptualización de la conciencia a partir de los planteamientos del eliminativismo y el funcionalismo y, en segundo lugar, realizar un análisis crítico con respecto a dichos planteamientos.

Ya sabemos, entonces, que una similitud, en rasgos generales, es que el eliminativismo y el funcionalismo pertenecen a la misma corriente: monismo psicofísico contemporáneo. Por otro lado, consideramos preciso aclarar lo siguiente y es que, dentro del monismo psicofísico contemporáneo: i) el materialismo eliminativista se presenta como una corriente reduccionista y ii) el funcionalismo se muestra como una corriente no-reduccionista. Por lo que aquí ya evidenciamos, entonces, la primera diferencia (Vega, 2017, pp. 52-61; Esparza-Oviedo, 2018, pp. 17-19, pp. 38-39).

Otra de las similitudes, que encontramos entre el materialismo eliminativista y el funcionalismo, es que ambas corrientes parten de la teoría de la identidad. Esta fue desarrollada a partir de los años cincuenta del siglo pasado y plantea, a grandes rasgos, que los estados mentales son idénticos a los estados del cerebro, por lo que se debe dar una reducción interteórica; esto es, debe existir una correlación en los relatos de las descripciones de los procesos neurales con los procesos mentales. Sobre los argumentos de la teoría de la identidad, veamos:

Sin ninguna duda apoyan en forma abrumadora la idea de que las causas de la conducta humana y animal son de naturaleza esencialmente física, pero la teoría de la identidad no se limita a afirmar sólo esto, sino que sostiene que la neurociencia ha de descubrir una taxonomía de los estados neurales que permita establecer una correspondencia biunívoca con los estados mentales de la taxonomía del sentido común: Las afirmaciones de identidad interteórica sólo quedarán justificadas si se puede encontrar esa correlación (Churchland, 1992, p. 54).

Pues bien, tanto el materialismo eliminativista como el funcionalismo parten de la teoría de la identidad ${ }^{4}$ como la fuente principal que les permite plantear y replantear los fenómenos cognitivos estrechamente ligados a los resultados

\footnotetext{
${ }^{4}$ Sobre la teoría de la identidad se recomienda revisar: Bello, 1979, pp. 67-86; Rorty, 1965, pp. 2528; Bechtel, 1991, pp. 127-137; Churchland, 1992, pp. 50-52; Esparza-Oviedo, 2018, pp. 18-21, pp. 23-24, pp. 36-37.
} 
de las ciencias empíricas, para este caso, de las neurociencias. No obstante, el materialismo y el funcionalismo se separan en el momento de refutar a la teoría de la identidad. Veamos, en la corriente del materialismo eliminativista surge la siguiente pregunta ipara qué admitir dos clases de descripciones de los fenómenos mentales si una sola cumple dicha función? Si con los avances de las neurociencias se ha venido dando respuesta al funcionamiento del sistema nervioso.

De acuerdo con la explicación ofrecida por Paul M. Churchland, el principal inconveniente de la teoría de la identidad se halla en la identificación de los estados mentales (1992, p. 56). Entonces, la manera en la que se refuta la teoría de la identidad se da como sigue: es necesario que una propiedad de un estado cerebral no coincida con un estado mental; por lo que, con respecto a las propiedades espaciales un estado mental debe encontrar una ubicación espacial determinada en un estado cerebral (Churchland, 1992, pp. 53-56; Botero, 1992, p. 68). En definitiva, para P. M. Churchland no es necesario recurrir a dos propuestas explicativas, pues con el avance de las neurociencias es suficiente para esclarecer asuntos como la enfermedad mental, la visión, el aprendizaje, entre otros. Y estas cuestiones no son más que las explicaciones necesarias sobre los orígenes de la conducta y los procesos cognitivos que permiten abordar y conocer el mundo (Churchland, 1992; 2001; 2002; 2006).

Por otra parte, el funcionalismo refuta la teoría de la identidad, pues que el funcionalismo al buscar un reduccionismo interteórico en donde los estados mentales se corresponden con un estado físico-cerebral específico, deja de lado que, en efecto, se encuentran diversos tipos de sistemas físicos "con economía funcional de estados de inteligencia consciente" (Esparza-Oviedo, 2018, p. 38). Es decir, el funcionalista no reduce los procesos mentales a determinados sistemas físicos (Martínez-Freire, 2001, p. 92; Vega, 2017, p. 53; Churchland, 1992, pp. 65-67). Hasta este punto, podemos sintetizar de la siguiente manera: i) para el materialismo eliminativista el gran avance de las neurociencias nos permitirá reducir todo a una cuestión de estados cerebrales en donde se llegarán a suprimir los conceptos propios de la psicología popular (Churchland, 1992, pp. 76-80); esto es, su desemejanza con la teoría de la identidad radica en que los fenómenos mentales no se reconocen en estados físicos, pues no es necesario recurrir a conceptos mentales, solo existen estados físicos y sobre estos se debe conceptualizar; ii) para el funcionalismo, que también parte de su crítica a la teoría de la identidad, de igual forma se aleja del materialismo eliminativista, pues sostiene que no se trata de reducir todo a un sistema físico, debido a que no solo la estructura material de la 'máquina' determina su función (Martínez-Freire, 2001, p. 92).

Ahora, demos paso a lo que creemos es la principal diferencia que podemos encontrar entre el materialismo eliminativo y el funcionalismo, a saber: el 
funcionalismo no reduce todo a estados físicos, es decir, no condiciona el funcionamiento cognitivo a una determinada estructura material. Mientras que el materialismo eliminativo se plantea como una corriente reduccionista que depura la aparente relación entre los fenómenos mentales y los fenómenos cerebrales; para el eliminativismo lo único certero sobre los procesos cognitivos son los resultados aportados por las neurociencias, es decir, esta es la base fundamental "para explicar el funcionamiento de los procesos cerebrales, los cuales, en últimas, explican los procesos más básicos de la realidad humana" (Esparza-Oviedo, 2018, p. 41).

Por tanto, el materialismo eliminativista propone depurar los términos propios de la psicología popular, pues no son más que conceptos vacíos sin ningún sustento científico. Por otro lado, el funcionalismo es una corriente materialista no-reduccionista que compara los estados mentales con el funcionamiento de dispositivos mecánicos y gracias a los trabajos de Putnam y Fodor ${ }^{5}$ se supone que el procesamiento de información de la mente humana es semejante al de una computadora: el computacionalismo como teoría funcional plantea al cerebro como un procesador simbólico en donde lo que importa es la función (software) no el funcionario (hardware) (Vergara, 2004, pp. 50-53; Lycan, 1995, p. 143).

En consecuencia, nos atrevemos a afirmar que esta principal diferencia entre el eliminativismo y el funcionalismo obedece a que este último plantea que para la comprensión de los estados cognitivos es necesario saber que estos operan de forma causal de acuerdo a inputs sensoriales y outputs conductuales que varían de acuerdo con los estados mentales mismos, los cuales también dependen del ambiente (Rabossi, 1995, pp. 24-25; Lycan, 1995, p. 143). Además, a esto agregamos que el funcionalismo no presenta como necesaria la idea de eliminar ciertos conceptos de la psicología popular, sino que muchos de estos son replanteados y otros depurados dependiendo de los resultados de la ciencia. Sobre los conceptos replanteados, tal es el caso mismo de la conciencia, tema sobre el cual estamos discurriendo en este trabajo.

Pues bien, con respecto a otra diferenciación entre el eliminativismo y el funcionalismo, apuntemos a la crítica directa del funcionalismo a las teorías reduccionistas, a partir de la explicación de los sistemas funcionales. Y, para ello, tengamos presente lo siguiente: un sistema es intencional si permite predecir el comportamiento de un organismo o un sistema, por lo que debe reunir tres características — de acuerdo con la lectura dennettiana de Botero-: i) un programa o cierta función, ii) composición física, y iii) cierta racionalidad. Ya podemos dilucidar aquí que el funcionalismo no es una corriente reduccionista, pues no restringe la actividad mental a una mera función material, por lo que "esto es lo que permite que Dennett en su obra intente abordar temas omitidos por otros

${ }_{5}^{5}$ Sobre los trabajos de Putnam y Fodor se recomienda revisar: Rodríguez, 2006 y Blanco, 2001. 
autores (quizá, considerados, de un perfil materialista radical o reduccionista), tales como: percepciones, creencias, sentimientos, etc." (Esparza-Oviedo, 2018, pp. 46-47). Como ya dijimos, en el funcionalismo no se reducen los estados mentales a estados físicos o no se eliminan de manera concluyente los términos propios de la psicología popular, sino que estos conceptos se depuran, se revisan y se replantean.

De la misma manera, adentrémonos en el punto que más nos importa con propiedad, y es encontrar las diferencias y las similitudes entre el materialismo eliminativo y el funcionalismo de acuerdo con sus respectivas conceptualizaciones sobre la conciencia. Comencemos por recordar que, en términos generales, el principal inconveniente de abordar el problema de la conciencia es su conceptualización misma, pues no hay acuerdo al respecto en las ciencias cognitivas. Tal como lo plantea Arias, el debate sobre la conciencia es decisivo en el desarrollo actual de las ciencias cognitivas, pero, lamentablemente, carecemos de un consenso mínimo sobre la misma (Arias, 2016, p. 65). Ahora bien, teniendo en cuenta a Thomas Nagel y partiendo del problema mente-cerebro acerca de la experiencia consciente, existe una profunda división entre quienes abordan esta problemática desde la perspectiva reduccionista y la no-reduccionista; es decir, entre quienes afirman que los fenómenos mentales pueden ser reducidos a procesos físicos y quienes piensan que los fenómenos mentales son diferentes de todo lo demás por lo que no se acotan a un sistema físico-material. Veamos lo que Nagel nos dice:

La más profunda división entre los que escriben sobre la mente y el cerebro se encuentra entre reduccionistas y antirreduccionistas: entre quienes piensan que, algún día, los fenómenos mentales podrán ser explicados con los recursos de la ciencia física, y quienes piensan que los fenómenos mentales son tan radicalmente diferentes de todo lo demás que se necesita una extensión igualmente radical de las formas de explicación científica para explicar cómo ellos surgen de la operación física del cerebro (2000, p. 112).

Pasemos, entonces, a la forma en la que se aborda la conciencia a partir del materialismo eliminativista. Consideremos la siguiente cuestión: si la conciencia de acuerdo a sus generalidades es un problema propio de la subjetividad, ¿cómo es posible abordarlo de manera científica? Por otra parte, recordemos que, de acuerdo con P. S. Churchland, la conceptualización de la conciencia no resulta problemática debido a que la ciencia, más que partir de definiciones, lo que propiamente realiza es ofrecernos dichas definiciones, a partir de los fenómenos abordados, con el propósito de ampliar nuestro conocimiento (Churchland, 2002, p. 133; Arias, 2016, p. 65). Y, por otro lado, con respecto a la cuestión de la conciencia como un fenómeno subjetivo que se intenta abordar de manera objetiva (científica), en rasgos generales, podría llegar a ser resuelta modificando 
el problema mente-cuerpo por el problema mente-cerebro (Arias, 2016, pp. 7274, p. 103; Lycan, 1996, p. 2). Por lo que los eliminativistas plantean que nuestras nociones mentales son producto de un marco teórico heredado culturalmente, asimismo, manifiestan que todos los términos mentalistas no son más que un flatus vocis. Entonces, no existe algo a lo que propiamente pueda denominarse como "conciencia": "el concepto de conciencia ni siquiera puede llegar a ser sustituido por otro "más operacionalizable", por lo que debe llegar a ser descartado con el resto de nociones mentalistas" (Esparza-Oviedo, 2018, p. 31). Veremos, entonces, que esta es la sustancial diferencia entre eliminativistas y funcionalistas, pues, para los primeros, todos los demás términos de la psicología popular deben llegar a ser eliminados; frente a esto P. M. Churchland afirma que existen los medios que permitirán prescindir de este lenguaje fosilizado y que la neurociencia asuma su función explicativa pertinente (1992, p. 82). Pero, al ser la conciencia uno de los fenómenos más complejos de abordar, los Churchland terminan por volver a este término, aunque de una manera ambigua. De allí que intenten explicar de manera depurada la conciencia a partir del marco de las neurociencias. No obstante, esta exposición sobre la conciencia queda inacabada; es más, los Churchland no son explícitos y claros al momento de sostener si aceptan o niegan definitivamente el problema de la conciencia. Además, cuando hacen referencia al concepto de conciencia, no explican propiamente qué es esta, sino algunas funciones cerebrales que serían propias de la conciencia, sin que podamos tener claro qué vendría siendo la conciencia para ellos (Churchland, 2001, pp. 259285; Esparza-Oviedo, 2018, pp. 32-35). No obstante, a pesar de esta ambigüedad en el discurso, la balanza se inclina más hacia la negación de la conciencia (Esparza-Oviedo, 2018) en la medida en que, tanto los estados mentales como la conciencia, terminan siendo definidos como patrones de actividad neuronal (Churchland, 2001; Esparza-Oviedo, 2018; Muñoz, 2015 y Churchland, 2006).

Por otra parte, tenemos la conceptualización sobre la conciencia que nos ofrece el funcionalismo de Daniel Dennett. Si bien los funcionalistas no reducen los fenómenos mentales a que dependan de una estructura física determinada, los fenómenos mentales son los efectos físicos de las actividades del cerebro; tal es el caso de la conciencia. Por lo que Dennett afirma que el gran misterio de esta problemática se debe a cómo es posible que los cuerpos físicos que habitan en un mundo físico sean capaces de producir tales fenómenos (Dennett, 1995, p. 37). Entonces, el reto es explicar la conciencia —en general, los estados mentales- en donde esta no debe ser entendida únicamente desde una perspectiva biológica (física) o una perspectiva cognitiva (diseño), sino también, "a partir de sus deseos, creencias, contexto social, etc., (intencionalidad)" (Esparza-Oviedo, 2018, p. 50).

Esta perspectiva de la intencionalidad permite que los fenómenos mentales no sean reducidos a una mera actividad neurológica (Dennett, 1991, pp. 1620). A partir de esto, no se indaga solo por los estados mentales propios, sino 
por los de los demás; por lo que surge la siguiente pregunta: ¿cómo es posible verificar estados intersubjetivos? La solución que ofrece Dennett al respecto es conocida como la interpretación en tercera persona que no es más que la unión de la perspectiva personal y las interpretaciones realizadas de los hechos, constituyendo, desde esta postura, una perspectiva científica. Es decir, en primera persona se actúa como en un monólogo (desde la experiencia propia) y en tercera persona se recogen los datos de los hechos o fenómenos exteriores con el fin de ser interpretados, pues no podemos saber con toda certeza qué ocurre en otras mentes (la imposible, al menos hasta el momento, verificación de la experiencia intersubjetiva como fenómeno físico). Para Dennett, entonces, no se aborda el fenómeno mental de manera directa a partir del testimonio del sujeto mismo, sino que, por la vía neutral y objetiva, se recogen los testimonios de los sujetos para ser interpretados por otros (Sánchez, 2014, p. 42; Dennett, 1995, pp. 82-91).

En consecuencia, podemos pensar el fenómeno de la conciencia como una dinámica colectiva, pues para Dennett los fenómenos mentales pueden ser transmitidos como relatos a los demás (Dennett, 1991, pp. 16-20). Y es aquí cuando Dennett termina de dar sustento a su planteamiento partiendo de la teoría memética de Richard Dawkins: son los memes unidades de información cultural que, al igual que los genes, transmiten información; pero para este caso, es información cultural que se hereda en el interaccionismo para poder pasar a afirmar que la conciencia es una creación de la evolución cultural (Hierro, 2005, p. 180; Sánchez, 2014, p. 43; Dawkins, 1993, p. 21; Dennett, 1995, pp. 213215).

En este apartado podemos afirmar con respecto a la conciencia que, si bien para el eliminativismo y el funcionalismo es fundamental tener en cuenta los procesos físico-cerebrales, para el eliminativismo es necesario limitarse al campo de las neurociencias, por un lado, mientras que el funcionalismo permite ser más amplio con respecto a la forma de abordar el fenómeno de la conciencia, no hay una mera reducción a fenómenos cerebrales, pues queda abierta la posibilidad de la interpretación. Entonces, el materialismo eliminativo, en su aspiración de depurar los conceptos propios de la psicología popular, en definitiva, cierra la puerta al fenómeno de la conciencia, pues en los casos en que se hace mención a dicho fenómeno su referenciación no es clara y directa, sino que discurre en divagaciones imprecisas con respecto a fenómenos neurobiológicos, pero no dice "esto es la conciencia" o "sí es posible abordar un fenómeno que tradicionalmente se ha caracterizado por ser propio de la subjetividad".

Recordemos lo que puede ser una contundente crítica desde el materialismo eliminativista al funcionalismo, pues, desde una postura naturalista, la noción de la experiencia consciente, como un fenómeno singular (subjetivo) que primero supone el conocerse a sí mismo para posteriormente conocer los fenómenos exteriores, es una construcción ficticia que debe ser descartada (Churchland, 
1992, pp. 68-74). Y, en desacuerdo con todo esto, el funcionalismo plantea que los cerebros humanos están conformados por memes que permiten estructurar una conciencia colectiva que es producto de una evolución cultural (Dennett, 1995, pp. 465-466; Sagan, 2015, p. 14).

\section{Análisis crítico acerca de los planteamientos del "materialismo eliminativista" y del "funcionalismo" con respecto a su conceptualización acerca de la conciencia}

En este apartado nos enfocaremos en los siguientes puntos de manera especulativa: i) el problema del reduccionismo biológico y sus implicaciones en la voluntad libre en relación con el materialismo eliminativo y ii) problematización del fenómeno de la conciencia planteado por el funcionalismo.

\subsection{El problema del reduccionismo biológico y sus implicaciones en la voluntad libre en relación al materialismo eliminativo}

Para iniciar este apartado nos parece pertinente hacer referencia a la crítica clara y precisa que realiza Botero con respecto a los reduccionismos y los determinismos — siendo la referencia a los reduccionismos biológicos el asunto en el que nos interesa profundizar aquí-, veamos: "Hoy en día, debe reconocerse, buena parte de la humanidad avanza a pasos agigantados pretendiendo demostrar que los hombres son objetos de un determinismo desmesurado, ya sea del orden psíquico, biológico, social o divino" (Botero, 1999, parágrafo 7).

Tal es el caso que Stephen Hawking considera que, con respecto al determinismo existen leyes que gobiernan y explican los fenómenos del universo. Y, aunque no hemos hallado la forma exacta de todas estas leyes, Hawking era optimista en considerar probable que se hallarán las leyes que determinen por completo la evolución del universo (1994, p. 136). Así, a partir de Hawking intentaremos aclarar nuestro panorama. Veamos:

En JULIO CÉSAR, la tragedia de Shakespeare, Casio le dice a Bruto: "Los hombres son a veces dueños de su destino". ¿Somos realmente dueños de nuestro destino? ¿O está ya determinado y preordenado todo lo que hacemos? El argumento en pro de la predeterminación solía señalar que Dios es omnipresente y se halla al margen del tiempo, de modo que sabe lo que va a suceder. ¿Cómo podemos tener entonces libre albedrío? ¿Cómo es posible, de no tenerlo, que seamos responsables de nuestras acciones? No podría ser culpable quien atracase un banco si estuviera predeterminado que lo haría. ¿Por qué castigarle? (Hawking, 1994, p. 135). 
Esta lectura de Hawking también es realizada por Botero y permite ir al planteamiento sobre el determinismo biológico, en donde expone que se pretende explicar la conducta humana a partir de los rasgos anatómicos o cerebrales del organismo dejando de lado "toda posibilidad de libertad en el hombre y, por ende, las posibilidades de elección" (Botero, 1999, parágrafo 7); que vendría siendo el caso del materialismo eliminativista. Dicho esto, vamos por partes. Indaguemos primero sobre eso qué es la libertad de elección, el libre albedrío o la libertad de la voluntad, para, después, contrastar esto con lo dicho desde una postura determinista y reduccionista, como lo es el materialismo eliminativista.

El tema del libre albedrío es quizá uno de los más problemáticos que se dan en el seno de la filosofía y la teología, pero que después se extrapola a diferentes disciplinas: neurociencia, psicología, derecho, antropología, entre otros. En donde pareciera que el libre albedrío es un enfrentamiento del individuo con la divinidad o la naturaleza, considerada esta como un algo determinista (Caro, 1988, p. 26). A grandes rasgos, el libre albedrío es la capacidad de elegir entre diferentes alternativas o de crear nuevos problemas sobre los cuales hay que escoger; en el caso de la perspectiva teológica cristiana se hace referencia a la posibilidad de elegir entre el bien y el mal (Bartra, 2013, p. 11):

La expresión «libre albedrío» proviene del latín «liberum arbitrium», muy usada por teólogos y filósofos cristianos, y se diferencia de la palabra «libertas» porque ésta se refería más al estado de bienaventuranza eterna, mientras que el libre albedrío se empleaba para designar la posibilidad de elegir entre el bien y el mal. San Agustín decía: «Debe confesarse que hay en nosotros libre albedrío para hacer el mal y para hacer el bien» (De corruptione et gratia, I, 2) (Rubia, 2003, p. 490).

Frente al tema complementemos con la siguiente cita:

podríamos entender como libre albedrío, a saber, que como ser humano:

(1) dispongo de un número, mayor que uno, de posibilidades entre las cuales escoger en mis decisiones y/o acciones, y (2) soy la fuente, el autor, el origen último de estas decisiones y/o acciones. Nuestro concepto preliminar de libre albedrío se basaría, en consecuencia, en dos condiciones fundamentales: (1) posibilidades alternativas de decisión y/o acción, y (2) control último sobre nuestras decisiones y/o acciones (Muñoz, 2015, p. 20).

De acuerdo con esto debemos decir que el problema del libre albedrío se encuentra ligado a la responsabilidad jurídica y moral ${ }^{6}$, si somos claros al entender por esta última la capacidad y obligación plena que tiene cada individuo de responder por sus actos; debido a esto los librealbedristas son realmente la

${ }^{6}$ Sobre la responsabilidad moral, véanse Patarroyo, 2019; Rosell, 2013; Ehman, 1963. 
contraparte de los deterministas, pues estos últimos sostienen que todos los eventos son producto de causas ya previas y para el caso de los deterministas reduccionistas todo se liga a causas físicas o materiales:

Parece entonces que si, por un lado, todo está determinado, que los hechos, sucesos o acontecimientos se desarrollan de acuerdo a leyes de la naturaleza que los prescriben; y que si, por otro, somos protagonistas y responsables de nuestros propios actos, entonces se enfrentan dos clases de conceptos incompatibles: «Las leyes de la física» y «la acción intencional». De manera que si se defiende la Libertad habrá de hacerse como una acción negadora del determinismo, de las leyes de la naturaleza (Pérez, 2008, p. 7).

Tal es el caso de los deterministas-reduccionistas, como los materialistas eliminativistas, que sostienen que el libre albedrío es una ilusión, no es más que una carencia, pues no se escoge nada, ya todo está fijo o establecido y lo que se debe hacer es encontrar la solución a los problemas que parecen más difíciles (Churchland, 2001, pp. 284-285). Consideremos que la postura librealbedrista se remite de manera directa al yo, a la mente y a la conciencia ${ }^{7}$, entendida esta postura como aquella relación que permite que el individuo pueda (re)pensarse a sí mismo y al entorno que le circunda (Welzel, 1973, pp. 221-230). Para el caso de los Churchland, si la conciencia es un mero concepto ilusorio, el libre albedrío también lo vendría siendo. Discurramos un poco al respecto, los Churchland suponen que algunos de los problemas tradicionales de la filosofía, tales como la naturaleza de la conciencia o la naturaleza del conocimiento, pueden llegar a ser explicados a cabalidad si realmente llegamos a entender el cerebro llevándonos a la idea, esencial y principal, de que únicamente somos materia; es decir, la mente es el cerebro y conceptos tales como la visión, la motricidad y demás, no son más que funciones del cerebro. Lo que nos lleva a afirmar que, en todos los casos en los que los Churchland hacen referencia a la conciencia, siempre están explicando funciones cerebrales como la percepción, el sueño, la conducta, y demás, dicho en otras palabras, el cerebro ni siquiera causa la conciencia, la conciencia es algún patrón de la actividad de las neuronas (Churchland, 2001, pp. 269-271), en donde la "potencia explicativa, la coherencia y la economía favorecerán la hipótesis de que la conciencia es justamente algún patrón de actividad de las neuronas" (p. 271). Veamos lo que Muñoz nos dice al respecto:

La neurofilosofía es la disciplina que analiza la repercusión filosófica de los descubrimientos de la neurociencia. Puede que el tema más importante que la neurofilosofía pretende abordar sea el problema mente-cuerpo, que nos invita a preguntarnos qué relación, si es que la hay, guardan los procesos nerviosos y los mentales. Estamos ante un problema clásico de la filosofía, y desde luego afecta directamente al debate sobre el libre albedrío si pretendemos estudiarlo desde una perspectiva neurofilosófica (2015, p. 82).

${ }^{7}$ Sobre la relación del libre albedrío con el yo, la mente y la conciencia, véanse: Ehman, 1963; Muñoz, 2015; Bartra, 2013, pp. 7-32. 
Con respecto a esto P. S. Churchland (2006), en el artículo The Big Questions: Do We Have Free Will?, hace referencia al caso de un hombre en Viginia (EE.UU.), quien sin ningún tipo de antecedentes comenzó escondiendo pornografía infantil y a abusar de su hijastra, una menor de ocho años de edad, posteriormente este hombre comenzó a quejarse de fuertes dolores de cabeza por lo que le enviaron un escáner cerebral en donde se mostró un tumor grande y benigno en el área frontal del cerebro, invadiendo parte del tabique y el hipotálamo, siendo estas las regiones que regulan el comportamiento sexual (Churchland, 2006, p. 42). Después de la extirpación del tumor sus intereses volvieron a lo que consideramos "normal"; sin embargo, al tiempo su propensión hacia la pedofilia volvió y otra exploración cerebral reveló que quedaron trozos de tejido después de la cirugía y que se había convertido nuevamente en un tumor considerable afectando, una vez más, su perfil conductual. En lo que respecta a esta exposición P. S. Churchland voltea su mirada a un problema en concreto y es el tema del libre albedrío, donde cuestiona si este individuo era responsable o no de su propia conducta:

This case renders concrete the issue of free will. Did the man have free will? Was he responsible for his behaviour? Can a tumour usurp one's free will? With the tumour, he had powerful, but atypical desires; he was not himself (Churchland, 2006, p. 42).

La respuesta frente dichas cuestiones es clara, al menos para la postura de los eliminativistas y lo que observamos, en este caso, de P. S. Churchland: el libre albedrío, al igual que la conciencia, es una mera ilusión. Estos no son más que conceptos propios de la psicología popular que deben ser depurados (Churchland, 1992, pp. 75-78; Bechtel, 1991, pp. 137-142; Arias, 2016, pp. 125-126). Tal es el caso, entonces, que las neurociencias y la biología nos están revelando y explicando gradualmente cómo funciona nuestro comportamiento (toma de decisiones y control de impulsos). Veamos:

Neuroscience, and behavioural biology more generally, are gradually revealing the mechanisms that make us who we are: how we make decisions and control our impulses, how our genes shape our social desires and how our reward system adapts in response to satisfying experiences (Churchland, 2006, p. 42).

En respuesta a esto, Roger Bartra afirma que los reduccionistas-deterministas han pasado por alto que la toma de decisiones no se encuentra restringida a un proceso físico —en este caso cerebral o neuronal—, sino que también depende del contexto sociocultural:

El contorno no es únicamente la fuente que nutre el proceso de aprendizaje ni es tampoco el contexto receptor que adapta los flujos generativos de los módulos neuronales. Una parte sustancial de este contorno está indisolublemente unida a la red neuronal, de la misma manera en que el 
biólogo Jakob von Uexküll definía el Umwelt, el universo subjetivo de un organismo donde cada componente tiene para él un significado funcional. El organismo opera con un conjunto de signos receptores y efectores que lo ligan a su propio Umwelt (2013, p. 58).

Para concluir este apartado, nos parece pertinente y necesario referirnos al planteamiento que realiza Bartra sobre el juego. Pues es preciso que pensemos el juego como un recurso que estimula los procesos simbólicos que se encuentran incompletos si reducimos la acción humana a meros procedimientos neuronales. Para Bartra, el juego es una actividad de orden y libertad en donde se impulsa a los individuos a explorar, de manera espontánea, multiplicidad de actividades por desarrollar. Dicho en otras palabras, el juego es condición para el brote de la libertad:

EL JUEGO es una de las actividades humanas que más ha sido asociada con la libertad. Cuando los humanos juegan, se ubican en un espacio peculiar donde se practican actividades que no parecen necesarias ni útiles y donde reina el libre albedrío. Los mejores estudios sobre el juego no han dejado de señalar que se trata de un comportamiento libre y aparentemente superfluo (2013, p. 72).

Es el juego una actividad libre-voluntaria que de manera simultánea implica un orden. Esta combinación, según Bartra, también implica actividades tales como la música, la danza, las artes plásticas, el deporte, entre otras. Entonces, ya hemos dicho que si bien el juego implica un orden, unas ciertas reglas, su foco principal es la libertad: iel libre juego de la fantasía!, que contribuye a construir un entorno práctico que ayuda a "cimentar las relaciones sociales y a afinar tanto la musculatura como el sistema nervioso" (Bartra, 2013, p. 75). Podemos inferir de esto que el juego carece de una utilidad inmediata porque no es más que la simulación de potenciales situaciones, veamos:

A mi juicio, el juego es una de las expresiones primordiales y acaso más puras de lo que he llamado la incompletitud del cerebro. El juego es una prótesis inútil en su expresión inmediata, pero contribuye a estimular los procesos simbólicos de sustitución (Bartra, 2013, p. 74).

\section{2. Problematización del fenómeno de la conciencia planteado por el funcionalismo}

Como ya sabemos, el fenómeno de la conciencia no es negado por la corriente funcionalista de Daniel Dennett. Al contrario de los eliminativistas, Dennett no pretende depurar los conceptos propios de la psicología popular, al menos no todos. Recordemos que los funcionalistas no interpretan los fenómenos mentales como una mera reducción de procesos que dependen de una estructura física 
determinada. Sabemos, entonces, que al momento de explicar la conciencia, Dennett plantea que esta no es entendida solo desde la perspectiva del diseño o la perspectiva física, sino también desde la intencionalidad (Dennett, 1995, p. 50). En consecuencia, no se indaga únicamente por los estados mentales que son propios, sino de los otros, lo que conlleva realizar una investigación intersubjetiva. La pregunta que nos surge con respecto a esto es: ¿cómo es posible verificar estados intersubjetivos? Dennett, como ya lo vimos, propondrá al respecto la interpretación en tercera persona que conlleva que el fenómeno de la conciencia sea pensando como el resultado de una dinámica colectiva producto de evolución misma (Sánchez, 2014, p. 42; Dennett, 1995, pp. 82-91); por lo que enlaza dicho planteamiento con la teoría memética de Dawkins: la transmisión de información cultural hereditaria (Dawkins, 1993, pp. 21-29; Dennett, 1995, pp. 213-215; Hierro, 2005, p. 180; Sánchez, 2014, pp. 42-43).

Dicho esto, nos parece pertinente detenernos un poco y realizar los siguientes cuestionamientos al respecto: si decimos "soy consciente de un objeto", ¿implica que puedo demostrar que el objeto existe? ¿Existe porque soy consciente de él? Con respecto a esto, diríamos que la respuesta desde una perspectiva funcionalista es sencilla, y es que, como ya dijimos, en primera persona se actúa de tal forma como si pareciera un monólogo, casi que nos atreveríamos a decir, como si fuera un soliloquio: aquel discurso que mantiene un individuo consigo mismo. Es decir, se ofrece un discurso desde la experiencia propia, lo cual nos parece absurdo, esto es porque se parte de una premisa de la cual se desconoce propiamente su verdad (en este caso, afirmar la existencia de "mi conciencia") para luego determinar mediante la concatenación de otras premisas toda la validez del argumento y afirmar la existencia de la conciencia a partir de un discurso en primera persona. Por lo que la pregunta nuevamente es icompruebo la existencia de mi conciencia porque soy consciente de que soy consciente?

Frente a esto, Dennett plantea que examinemos el asunto desde el punto de vista científico que él propone y es que dicho discurso científico se da en tercera persona. Así las cosas, bien sabemos que, desde esta postura funcionalista, la conciencia es una colectividad. Por tanto, en el análisis en tercera persona se recogen los datos proporcionados por otros para ofrecer una descripción más objetiva de la experiencia del mundo, que en primera instancia es interpretado desde la interioridad de un individuo (primera persona). Sin embargo, zesto es suficiente para poder afirmar que un determinado objeto existe o que esta sea la certeza necesaria para afirmar la existencia de algún fenómeno? Tengamos en cuenta que, esa tercera persona es elaborada por una primera persona, de modo tal que el yo subjetivo piensa colectivamente, pero sigue siendo en primera persona. Además, traigamos a colación, por hacer referencia a algunos de tantos casos posibles, aquellas situaciones con respecto al consumo de sustancias 
piscoactivas $^{8}$, que ejercen efectos directos en el sistema nervioso central (SNC), afectando nuestra capacidad de pensamiento racional:

La psiquiatría conoce cientos de productos químicos que controlan las emociones (litio, clorpromacina, [sic] anfetamina, cocaína y otros) que producen sus efectos cuando penetran en el cerebro. Y la vulnerabilidad de la conciencia frente a los anestésicos, la cafeína, y frente a algo tan simple como un fuerte golpe en la cabeza, demuestra su dependencia muy estrecha de la actividad nerviosa del cerebro. Todo esto tiene sentido si la razón, la emoción y la conciencia son actividades del cerebro mismo (Churchland, 1992, pp. 43-44).

Así que, con referencia a lo dicho, queda abierta la duda sobre cómo es posible ser consciente de un objeto o un determinado fenómeno, si en el caso del consumo de sustancias psicoactivas estas pueden frustrar o exagerar las actividades químicas que tendrán sus efectos sobre la comunicación nerviosa (Churchland, 1992, p. 209). Pues el consumo de estas sustancias psicoactivas puede producir unos efectos que "se asemejan mucho a los de las formas principales de enfermedades mentales: depresión, manía y esquizofrenia" (1992, p. 210). Lo que evidentemente afectaría la conducta y la percepción de un individuo sobre su mundo circundante; esto es, la tercera persona.

Ahora bien, si afirmamos "soy consciente de mí mismo", ¿es esta la prueba de la existencia de mi conciencia?, ¿cuál es la prueba de que soy consciente de mí mismo?, ¿por qué soy consciente de mí mismo?, es decir, ¿soy consciente de que soy consciente porque soy consciente? Esto no constituiría más que una petición de principio (petitio principii), falacia que también es conocida como el razonamiento circular que consiste en dar por sentado aquello que se pretende probar (Copi y Cohen, 2007, pp. 137-138). Por tanto, dicho planteamiento se nos convertiría en una reducción al absurdo, pues, si para suponer mi consciencia del mundo circundante, necesito de la interpretación de un tercero que diga algo sobre mi conciencia entonces, ¿cómo puedo decir que soy consciente de algo? A esto agreguemos que el cerebro puede construir falacias e ideas absurdas, decir que soy consciente de mí mismo no prueba nada.

Finalmente, nos queda por decir que la propuesta de Dennett, acerca de que la conciencia es producto de un proceso evolutivo cultural, sigue siendo una idea controvertida, pues de alguna forma reduce al ser humano a un simple dispositivo que se encuentra programado: "el ser humano no es más que un compuesto de

\footnotetext{
${ }^{8}$ Dentro de las sustancias más comunes, es decir, usadas de manera global encontramos el alcohol y el tabaco, es decir, son sustancias que pueden conseguirse, en la mayoría de los casos, de manera legal y en la mayor parte del mundo. Entre otras sustancias encontramos los opioides, los canabinoides, la cocaína, las anfetaminas, el éxtasis, los alucinógenos. Para tal caso, revisar Organización Mundial de la Salud, 2005.
} 
pequeños sistemas intencionales organizados según los propósitos de la evolución, propósitos que por otra parte son ciegos" (Colomina, 2004, p. 247).

Esto, por supuesto, obviaría la capacidad de los seres humanos de saberse libres sobre sus propios actos. Sabemos que Dennett retoma los postulados de Darwin a través de la lectura dawkinsiana de la evolución en donde si bien la evolución se da de manera aleatoria, no quiere decir que esta no tenga un orden, simplemente este orden no es perceptible para nosotros. Aunque para Dennett el ser humano es intencional -esto es, lo relativo a los estados mentales, deseos, creencias y demás-, el problema surge cuando se intenta ajustar la tesis de la intencionalidad con la propuesta dennettiana de que los seres humanos también somos un compuesto físico. Entonces, ¿es la conciencia una cuestión ilusoria? Según la lectura de Colomina:

En realidad el ser humano no es libre de actuar libremente de este modo porque no elige sus propias acciones: sólo responde a la configuración que realiza por él su propia historia cognitiva, lo que restringe su ámbito de acción. El desarrollo se produce de modo ciego pero no de modo desordenado (Colomina, 2004, p. 248).

Volvemos, entonces, al problema del libre albedrío, ¿estamos o no estamos predestinados? Recordemos la idea tradicional acerca del libre albedrío en la que se afirma que el ser humano es libre porque tiene la capacidad de causar sus propias acciones. Por otra parte, el desarrollo ciego de los organismos no es un desarrollo desordenado, pues el movimiento aleatorio de su adaptación al entorno se produce por ensayo y error, dependiendo de la forma en la que el organismo se relaciona con el medio ambiente. Por tanto, el problema del libre albedrio vendría siendo una ilusión, pues si bien la evolución no se encuentra determinada por leyes que son reduccionistas, al menos desde la lectura que Dennett hace sobre Dawkins, todo organismo sigue estando limitado al principal interés de la evolución que es la supervivencia y por ello los organismos se modifican o auto-modifican de acuerdo al modelo que ya tienen codificado en su genética. Y, para el caso de la teoría memética, los individuos ya tienen una pre-condición cultural —además de la genética-, por cual no es posible hablar de un libre albedrío, al menos en términos absolutos, de ahí que se presuma que es una ilusión (Colomina, 2004, pp. 247-250).

\section{Conclusiones}

El problema de la definición y la descripción acerca de la conciencia es abordado de manera aproximativa, pues no existe ni un relativo consenso en el área de las ciencias cognitivas. Debido a esto, lo que hemos planteado con certeza es que la conciencia es la reformulación del problema mente-cuerpo 
por el problema mente-cerebro. En consecuencia, entre las conclusiones más importantes resultado de nuestro trabajo consideramos destacar las siguientes:

Demos cuenta, entonces, de lo que es la conciencia tanto para el eliminativismo de los Churchland como para el funcionalismo de Dennett. Pues bien, para el materialismo eliminativista de los Churchland, corriente monista psicofísica contemporánea de corte reduccionista, los procesos cognitivos no son más que actividades de las neuronas, lo único que existe son los fenómenos físicos. El mundo físico es el único nivel de análisis objetivo en donde "realidad" y "materia" son términos coextensivos. De ahí que el eliminativismo guarde una estrecha relación con las neurociencias, por lo que P. M. Churchland plantea que, con el gran avance de estas ciencias en el futuro no será necesario recurrir a descripciones biunívocas como es el caso de la teoría de la identidad. Entonces, la neurociencia es la base fundamental del materialismo eliminativista propuesto por los Churchland para depurar los términos de la psicología popular y de la filosofía. Pues bien, frente al caso de la conciencia como un fenómeno que se presenta como subjetivo, en primera instancia, los Churchland plantean que aquellas nociones que conservamos acerca de las nociones mentales son producto de un marco cultural heredado, el cual pierde toda objetividad. En consecuencia, no existe algo que pueda ser denominado como conciencia, concepto el cual no puede llegar a ser reemplazado por otro más operacionalizable. La conciencia es un problema indescifrable, de tal manera que, cuando en las investigaciones realizadas por los eliminativistas se hace referencia al concepto de "conciencia", se tiene como finalidad que este sea negado o se toma para explicar ciertas funciones como el sueño, la comunicación neuronal, la función del sistema visual, entre otros. Es más, cuando se hace referencia a la conciencia, los Churchland no son lo suficientemente claros, divagan acerca del término sin llegar a reconocer su existencia; únicamente se limitan a explicar procesos cerebrales demarcados en el campo de la neurociencia.

Además, frente a esta definición eliminativista de la conciencia, se abre una discusión acerca del problema del libre albedrío, en la medida que el reduccionismo biológico de los Churchland pretende demostrar que estamos sujetos a un determinismo. Sobre esta postura eliminativista, que niega el libre albedrío, nos preguntamos si acaso esto no acarrea unas consecuencias políticas y sociales $^{9}$, pues tal como lo plantea Bruckner, a partir de la primera mitad siglo XX creamos y compartimos un ambiente de irresponsabilidad al desculpabilizarnos y esto se debe, en gran medida, a la interpretación de los resultados de las ciencias

\footnotetext{
${ }_{9}^{9}$ En general, con respecto a la negación del libre albedrío a partir de tales discursos reduccionistas y, por tanto, deterministas, sería pertinente indagar por las posibles consecuencias en el campo de lo que es la filosofía práctica: derecho, política, ética y moral. Este es un debate álgido en la actualidad, dados los avances y planteamientos hipotéticos en campos como las neurociencias, la inteligencia artificial, el transhumanismo, entre otros. Por lo que, es una discusión que debe ser abordada, cada vez más, con la profundidad que amerita el asunto.
} 
empíricas. La desculpabilización justificada a partir de las ciencias empíricas ha contribuido a que nos pensemos de esta manera: "ipara qué inventarme puesto que una «ciencia» me dice lo que soy y lo que seré, haga lo que haga (cosa en la que la sociología es tan descriptiva como prescriptiva)?" (1996, p. 39). Todo lo hasta ahora mencionado conlleva una negación de la responsabilidad, es una negación del deber lo que lleva al individuo a entregarse sin cuestionamiento alguno ante el fatalismo del destino. Por lo que, nos preguntamos iqué debe hacer la filosofía frente al afán de creernos determinados? Botero plantea la importancia del concepto de "visión responsable", el cual exige una posición y acción frente al otro y frente a lo otro como una forma de enfrentar los determinismos que nos desresponsabilizan (2002, pp. 18-27). En general, la cuestión de la responsabilidad juega un papel decisivo en el terreno de la filosofía, pues de manera directa se enfrenta a los determinismos y reduccionismos propios de las ciencias empíricas: es el sujeto quien se construye de manera individual y colectiva (Vanney, 2009, pp. 425-440; Cruz, 2000, pp. 15-26; Ströker, 1999, pp. 713-737).

Por otra parte, para el funcionalismo de Daniel Dennett, corriente psicofísica contemporánea considerada no-reduccionista, los conceptos mentales no se corresponden a un único tipo de estado físico, es decir: dentro de la multiplicidad existente de estados mentales también existe diversidad de sistemas físicos. Esto no quiere decir que el funcionalismo descarte la realidad material, pues también se asume como necesaria la percepción sensorial del mundo para poder conocerlo. Para el funcionalismo, los estados mentales se producen debido a su rol causal en donde existen inputs sensoriales y outputs conductuales que dependen de la variedad de estados mentales y sus posibles combinaciones. Además, estos estados mentales se encuentran condicionados por el ambiente el cual configura dichos estados.

De acuerdo con esto, Dennett sostiene que la mente humana no se configura solo por su perspectiva biológica y cognitiva, sino también desde la intencionalidad (creencias, deseos, perspectivas, entre otras). De modo que no importan únicamente los propios pensamientos, sino los de los demás, pues de esta manera es que los estados mentales podrán ser comprendidos. Es decir, la conciencia es una colectividad y para que los propios pensamientos puedan ser transmitidos y traducidos a un lenguaje científico es preciso que los hechos sean narrados en tercera persona con el propósito de proporcionar objetividad a la interpretación a realizar. Dennett complementa este planteamiento con la teoría memética de Richard Dawkins: los memes son unidades de información cultural que son transmitidas tal como sucede con los genes.

Por tanto, la conciencia es colectiva y es producto de la evolución cultural. Sin embargo, esta narración del yo en tercera persona sigue estando en la subjetividad, pues es una primera persona - que narra en tercera- quien da cuenta de la 
conciencia del otro. Entonces, si en el análisis en tercera persona se toman los datos acerca de los estados mentales, los cuales son proporcionados por otros para ser analizados, ¿esto es condición suficiente para suponer la existencia de un objeto o de la conciencia misma? De ser así, ¿̇tengo conciencia de mí mismo por otro?, ¿cómo se puede conciliar la interpretación subjetiva de diferentes fenómenos para ofrecer una descripción científica y objetiva? Como una suerte de contestación a este asunto, nos parece preciso decir lo siguiente: la ciencia misma es realizada por humanos y si asumimos la subjetividad como algo propenso a la imprecisión, entonces, la ciencia, en consecuencia, también lo será, pues es un constructo colectivo humano en movimiento permanente.

Pero, volviendo al tema de la conciencia, queda abierto el debate sobre la voluntad en el caso del consumo de sustancias psicoactivas, las cuales alteran los estados cognitivos y afectan de manera directa el sistema nervioso central; tema que, por cierto, en su contexto es delicado, pues se puede correr el riesgo de estigmatizar e infantilizar al consumidor de este tipo de sustancias y de judicializarlo al considerarlo un inimputable que requiere ser intervenido penal o clínicamente. Cerremos este punto diciendo que, la teoría memética se mantiene como una propuesta polémica en la que los seres humanos compuestos como sistemas intencionales se encuentran determinados a los propósitos de la evolución, propósitos que son ciegos.

Finalmente, sobre identificar las principales diferencias y similitudes acerca de la conceptualización de la conciencia entre la teoría materialista eliminativa y la teoría del funcionalismo, detallaremos los principales puntos. Tanto el materialismo eliminativista de los Churchland como el funcionalismo desarrollado por Dennett se presentan como corrientes que parten de la teoría de la identidad, pero en el transcurso de su análisis y crítica se separan. Por una parte, para los eliminativistas es innecesario admitir dos clases de descripciones de los fenómenos mentales cuando una sola cumple la función, que en este caso sería el discurso de las neurociencias: todo se reduce a estados cerebrales. Por otra parte, para los funcionalistas, el reduccionismo interteórico de la teoría de la identidad deja de lado que existe una diversidad de sistemas físicos y de estados mentales en los que existe una variedad de combinaciones para pasar a otro estado mental. Entonces, esta es la principal diferencia entre las dos corrientes; resaltando que los eliminativistas son reduccionistas, mientras que los funcionalistas no lo son. Esto nos hace suponer que los primeros niegan de manera contundente la conciencia y cuando hacen referencia a tal concepto lo realizan de manera negativa para decir lo que no es la conciencia, con el fin de no aceptar su existencia; mientras que los segundos dejan abierta la posibilidad de indagar por la misma. No obstante, encontramos como principal similitud que, en el momento de abordar la conciencia tanto desde el funcionalismo como desde el eliminativismo, es fundamental tener en cuenta los procesos físico-cerebrales. Entonces, lo que nos 


\section{Similitudes y diferencias en la conceptualización de la conciencia ofrecida por el materialismo eliminativo y el funcionalismo. Un análisis crítico}

parece importante decir es que sabemos bien que uno de los principales retos al abordar el problema de la conciencia no es solo la falta de una conceptualización más clara al respecto, sino la abundante cantidad de información que hay con referencia al tema y que pareciera no conciliarse desde las diferentes perspectivas, corrientes y disciplinas en las que se aborda.

\section{Referencias}

Alemañ, R. (2013). Dos caras de la misma moneda: mente, materia y monismo neutral. Naturaleza y libertad. Revista de estudios interdisciplinares, 2(1), 1132.

Arias, A. (2012). Del problema mente/cuerpo al estudio de la mente-cerebro. A partir de la neurociencia cognitiva. Thémata. Revista de Filosofía, (46), 249257.

Arias, A. (2016). El problema de la conciencia en la filosofía de la mente y las ciencias cognitivas: replanteamiento del núcleo del debate y valoración crítica de los principales marcos teóricos. (Tesis doctoral). Universidad Complutense de Madrid, Madrid, España.

Bartra, R. (2013). Cerebro y libertad. Ensayo sobre la moral, el juego y el determinismo. Ciudad de México: Fondo de Cultura Económica.

Bechtel, W. (1991). Filosofía de la mente. Una panorámica para la ciencia cognitiva. (L. Valdés, trad.). Madrid: Tecnos.

Bello, S. (1979). La tesis de la identidad entre estados mentales y estados fisicoquímicos. Diánoia, 25(25), 67-86.

Blanco, A. (2001). El funcionalismo y las ciencias cognitivas. Comentario A P. F. Martínez-Freire: "Base empírica y teoría funcionalista en las ciencias cognitivas". Ágora. Papeles de filosofía, 20(1), 105-112.

Botero, A. (1999). El secuestro como conducta atentatoria contra la libertad individual. (Tesis de pregrado). Universidad Pontificia Bolivariana, Medellín, Colombia.

Botero, A. (2002). El papel del intelectual: pasado, presente y futuro inmediato. Medellín: Editorial USB.

Botero, J. (1992). Algunas tendencias en la filosofía de la mente. Ideas y Valores, 41(87-88), 60-88. 
Bruckner, P. (1996). La tentación de la inocencia. (T. Kauf, trad.). Barcelona: Anagrama.

Caro, J. (1988). Releyendo textos sobre libre albedrío y la libertad. Eguzkilore: Cuaderno del Instituto Vasco de Criminología, (Extra 1), 17-28.

Churchland, P. S. (2001). ¿Puede la neurobiología enseñarnos algo sobre la conciencia? Contrastes. Revista Internacional de Filosofía, Suplemento VI, 259-289.

Churchland, P. S. (2002). Brain-Wise: studies in neurophilosphy. Cambridge: Bradford Books/The MIT Press.

Churchland, P. S. (2006). The big questions: Do we have free will? New Scientist, (2578), 42-45.

Churchland, P. M. (1992). Materia y conciencia. Introducción contemporánea a la filosofía de la mente. (M. Mizraji, trad.). Barcelona: Gedisa.

Colomina, J. (2004). El papel de la evolución en la teoría de Daniel Dennett. Arbor: Ciencia, pensamiento y cultura, CLXXIX(705), 247-264.

Copi, I. y Cohen, C. (2007). Introducción a la lógica. (E. González, trad.). Ciudad de México: Limusa.

Cruz, M. (2000). Los filósofos y la responsabilidad moral. Anuario de la Facultad de Derecho de la Universidad Autónoma de Madrid, (4), 15-26.

Dawkins, R. (1993). El gen egoísta. Las bases biológicas de nuestra conducta. (J. Robles, trad.). Barcelona: Salvat Editores.

Dennett, D. (1991). La actitud intencional. (D. Zadunaisky, trad.). Barcelona: Gedisa.

Dennett, D. (1995). La conciencia explicada. Una teoría interdisciplinar. (S. Balari, trad.). Barcelona: Paidós.

Ehman, R. (1963). La responsabilidad moral y la naturaleza del yo. Ideas y Valores, 5(18), 133-138.

Esparza-Oviedo, S. (2018). La conceptualización de la conciencia ofrecida por la "teoría del materialismo eliminativo" y la "teoría del funcionalismo", dentro del monismo psicofísico contemporáneo. Un análisis crítico. (Tesis de Maestría). Universidad Industrial de Santander, Bucaramanga, Colombia. 
Hawking, S. (1994). Agujeros negros y pequeños universos y otros ensayos. Ciudad de México: Planeta.

Hierro, J. (2005). Filosofía de la mente y de la Ciencia cognitiva. Madrid: Akal.

Lycan, W. (1995). La continuidad de niveles en la naturaleza. En E. Rabossi (comp.), Filosofía de la mente y ciencia cognitiva (pp. 143-170). Barcelona: Paidós.

Lycan, W. (1996). Consciousness and Experience. Cambridge: A Bradford Book/ The MIT Press.

Martínez-Freire, P. (1999). El debate mente-cerebro a la luz de las nuevas técnicas de exploración del cerebro. Contrastes. Revista Internacional de Filosofía, 4, 65-75.

Martínez-Freire, P. (2001). Base empírica y teoría funcionalista en las ciencias cognitivas. Ágora. Papeles de filosofía, 20(1), 87-104.

Muñoz, J. (2015). Libre albedrío y responsabilidad moral: una aproximación desde la neurociencia. (Tesis doctoral). Universidad Nacional de Educación a Distancia, España.

Nagel, T. (2000). Otras mentes. Ensayos críticos 1969-1994. (S. Girón, trad.). Barcelona: Gedisa.

Organización Mundial de la Salud (2005). Neurociencia del consumo y dependencia de sustancias psicoactivas. Washington: OPS.

Patarroyo, C. (2009). Libertad, determinismo y responsabilidad moral. Ideas y Valores, 58(141), 5-10.

Pérez, F. (2008). Sobre determinismo y libre albedrío. Eikasia: revista de filosofía, (16), 1-37.

Rabossi, E. (1995). Cómo explicar lo mental: cuestiones filosóficas y marcos científicos. En E. Rabossi (comp.), Filosofía de la mente y ciencia cognitiva (pp. 17-39). Barcelona: Paidós.

Rodríguez, M. (2006). Desmontando la máquina: las razones de Putnam contra el funcionalismo. Logos. Anales del Seminario de Metafísica, 39, 53-76.

Rorty, R. (1965). Mind-Body Identity, Privacy, and Categories. The Review of Metaphysics, 19(1), 24-54. 
Rosell, S. (2013). Voluntad y responsabilidad moral. Revista de Filosofía, 38(1), 121-138.

Rubia, F. (2003). ¿Existe el libre albedrío? Anales de la Real Academia Nacional de Medicina, (3), 489-509.

Sagan, C. (2015). Los dragones del Edén. Especulaciones sobre la evolución de la inteligencia humana. (R. Andreu, trad.). Barcelona: Crítica.

Sánchez, A. (2014). La concepción del yo en Daniel Dennett: un análisis de la relación entre la perspectiva heterofenomenológica y el enfoque memético. Logos: Revista de Lingüística, Filosofía y Literatura, 24(1), 40-50.

Ströker, E. (1999). La ética de la responsabilidad como desafío de la filosofía en el mundo moderno. Areté, XI(1-2), 713-737.

Vanney, M. (2009). Libertad, responsabilidad y sentido común en el pensamiento de Álvaro D’ors. Thémata. Revista de Filosofía, (41), 425-440.

Vega, S. (2017). Funcionalismo, reduccionismo y antirreduccionismo en la filosofía de la mente (II). Hojas Universitarias, (55), 52-61.

Velmans, M. (2009). Understanding Consciousness. New York: Routledge.

Vergara, P. (2004). Ser o no ser... individualista: ¿un dilema? Argumentos para el (anti)individualismo en Psicología. (Tesis de maestría). Universidad de Chile, Santiago de Chile. Recuperado de http://repositorio.uchile.cl/bitstream/ handle/2250/108799/Ser-o-no-ser-individualista-un-dilema.pdf?sequence = 4

Welzel, H. (1973). Reflexiones sobre el libre albedrío. Anuario de derecho penal y ciencias penales, 26(2), 221-230. 